\title{
A full rate concatenated space-frequency and space-time OFDM over Naftali fading channels
}

\begin{abstract}
In this paper we investigate 2 transmit and 1 receive (2Tx:1Rx) space-frequency orthogonal frequency division multiplexing (SF-OFDM) and space-time OFDM (ST-OFDM) concatenated with convolutional channel coding as a redundancy method to combat channel impairments. We built our SF and ST-OFDM on top of our coded OFDM (COFDM) with coding rate of $1 / 4$ and constraint length of 10 . We simulate our concatenated SF-OFDM and ST-OFDM over a multipath fading channel using Naftali 802.11 channel model under different delay spreads in relation to outdoor environment. Concatenated SF- OFDM performs slightly better than ST-OFDM at delay spread of 2.0 microseconds, but both are almost comparable at all other delay spreads. These two schemes outperforms other schemes such as single antenna coded OFDM and Alamouti's Space-Time Block Code (STBC) under these conditions by as much as $10 \mathrm{~dB}$ at BER of 104 .
\end{abstract}

Keyword: Space-frequency; Space-time; OFDM; Naftali; Alamouti's Space-Time Block Code (STBC) 